\title{
Group-Theoretical Foundations of Classical Mechanics: The Lagrangian Gauge Problem
}

\author{
Jean-Marc Levy-Leblond \\ Physique Théorique, Nice* \\ Received October 21, 1968
}

\begin{abstract}
This paper is devoted to the study of the classical, single and free particle...
\end{abstract}

\section{Introduction}

Invariance principles nowadays have become one of the most useful concepts of theoretical physics, mainly because of their importance in quantum theories. Indeed, when the dynamical laws obeyed by physical systems are not known or poorly understood, invariance principles act as "super-laws" to restrict the possible forms of these laws, as guides to find them [1]. Conversely, it may be argued that the use of invariance principles becomes obviated once the laws of nature are known, as is the case at the classical level. However, it seems to me that even then, invariance principles keep a prominent role in that they enable us to reach a deeper understanding of these (known) laws, reduce some of their apparent arbitrariness, relate previously unconnected concepts. The tighter structure and greater unity thus obtained have both an epistemological and pedagogical significance.

From that point of view, it is a striking fact that some very basic problems concerning the use of invariance principles in Classical Mechanics have not yet been studied, in direct contrast with the analogous problems in Quantum Mechanics. This paper is devoted to the solution of one of these problems which I will now state.

In Lagrangian Classical Mechanics, as is well-known, the Lagrangian of a given system is not uniquely determined. One can add to it the total time derivative of any function of the system variables, without modifying the equations of motion. Let us call "gauge transformations" such transformations, since the standard gauge transformation of electromagnetism is a special one. The point now is the following: the Lagrangian for a free relativistic particle is invariant under the Poincare group and may be derived from the assumption of invariance [2]. But why should a transformation of the Poincaré group leave invariant the Lagrangian, and not amount more generally to a gauge transformation? Indeed, the Lagrangian for a free non-relativistic particle is not invariant

\footnotetext{
* Equipe de Recherche Associée au C.N.R.S.
} 
under the Galilei group: pure Galilei transformations induce a gauge transformation [3]. If one wants to derive rigorously the dynamics of a free particle from the invariance group (Poincaré or Galilei for instance), it must be known precisely whether or not one can get rid of the possible gauge transformations by a suitable redefinition of the Lagrangian. Of course, one may derive directly the possible motions of a free particle without using the Lagrangian method, since they belong to the appropriate invariance group considered as a group of active transformations. However, the knowledge of the free Lagrangian is necessary when one needs introducing interaction with an external field ${ }^{1}$. Furthermore, one of our results precisely will be that some Lagrangians may describe more general motions than the ones belonging to the kinematical group under which they gauge-transform. A hint towards the solution of this problem is given by the answer to the analogous question in Quantum Mechanics; to a quantum system, there corresponds a unitary representation of the invariance group which need not be a vector representation, but may be a projective, i.e. up-to-a-factor, representation [4]. In such a case, the phase of the wave-function will be changed under a group transformation. Since in the classical limit, the phase of the wave-function goes into the classical action, we are led to foresee some relation between the classical and the quantum problems. This analogy becomes even more suggestive when we notice that for the nonrelativistic free particle, the classical action [3] and the phase of the Schrödinger wave-function $[4,5,7]$ transform identically under a galilean transformation. Indeed, our general conclusion will be that the possible transformation laws of the classical action under the invariance group, are closely connected to the projective representations of the group.

It should be mentioned that in the Hamiltonian form of Classical Mechanics, it may be shown that the Poisson brackets of the infinitesimal generators of symmetry transformations must realize a projective representation of the Lie algebra of the invariance group [8]. However, the canonical formalism seems to be a derived construction, and the immediate approach to Classical Mechanics, closer to one's intuition, is to consider configuration space and velocities rather than phase space and momenta, that is the Lagrangian method, which is the subject of the present investigation. Let us also mention, as related to our study, the interesting papers by TASSIE and BUCHDAHL [9], in which they show how to generalize the usual derivation of conservation laws to a theory where the Lagrangian is gauge-transformed under the invariance group,

${ }^{1}$ A very similar situation exists in the quantum theory, where the unitary representations of the invariance group are quite sufficient to study non-interacting systems, which one nevertheless tries to describe by means of wave-equations, in order to be able to introduce the effect of interactions with external fields [6].

5 Commun.math. Phys., Vol. 12 
with emphasis on the case of a single particle in special electromagnetic fields. Naturally, they start from a given Lagrangian, while we want to show how to derive it while allowing for gauge-variance.

Section 2 contains some definitions and propositions concerning exponents of groups ${ }^{2}$, which are used in Section 3 to obtain a grouptheoretical characterization of gauge-transforming Lagrangians. Section 4 contains a number of illustrative examples, which motivate the concluding remarks of Section 5 .

\section{Group Exponents and Superequivalence ${ }^{2}$}

The notion of exponents of a group $G$ has been introduced and extensively studied by BARGMANN in his work on ray representations of continuous groups [4]. We will reproduce here the definition of exponents and explore some further properties which will be used in the following section. For motivations, fundamental results and proofs in the theory of exponents, the reader is referred to Bargmann's paper.

\section{$2 a$}

Definitions [4]. An exponent of a topological group $G$ is a real valued continuous function $\xi$ defined on $G \times G$ and satisfying:

$$
\left.\begin{array}{c}
\xi\left(g_{3}, g_{2} g_{1}\right)+\xi\left(g_{2}, g_{1}\right)=\xi\left(g_{3}, g_{2}\right)+\xi\left(g_{3} g_{2}, g_{1}\right) \quad\left(g_{1}, g_{2}, g_{3} \in G\right), \\
\xi(e, e)=0 \quad(e=\text { neutral element of } G) .
\end{array}\right\}
$$

It follows from (1) that $\xi(e, g)=\xi(g, e)=0, \forall g \in G$.

Two exponents $\xi$ and $\xi^{\prime}$ of $G$ are called equivalent if there exists a real valued continuous function $\zeta$ on $G$ such that the following equivalence relation holds:

$$
\xi^{\prime}\left(g_{2}, g_{1}\right)=\xi\left(g_{2}, g_{1}\right)+\zeta\left(g_{2}\right)+\zeta\left(g_{1}\right)-\zeta\left(g_{2} g_{1}\right) .
$$

Due to the linearity of the defining relation, the exponents of a group $G$ form a real vector space. The equivalence class of a linear combination of exponents depends only on the equivalence classes of the exponents. It follows that the equivalence classes of exponents of a group $G$ form a real vector space.

\section{$2 b$}

Proposition 1. The equivalence classes of exponents of $G$ are invariant under the inner automorphisms of $G$.

Proof. Given an exponent $\xi$ of $G$ and the inner automorphism characterized by $g \in G$, call

$$
\xi_{g}\left(g_{2}, g_{1}\right)=\xi\left(g g_{2} g^{-1}, g g_{1} g^{-1}\right) .
$$

2 This section might also be used as an Appendix by readers who would not realize its interest - under the condition, of course, that they find this interest in the following section. 
Then a repeated use of the defining relations (1) yields:

$$
\xi_{g}\left(g_{2}, g_{1}\right)=\xi\left(g_{2}, g_{1}\right)+\zeta_{g}\left(g_{2}\right)+\zeta_{g}\left(g_{1}\right)-\zeta_{g}\left(g_{2} g_{1}\right),
$$

where $\zeta_{g}\left(g^{\prime}\right)=\xi\left(g^{-1}, g g^{\prime} g^{-1}\right)-\xi\left(g^{\prime}, g^{-1}\right)$, so that $\xi$ and $\xi_{g}$ belong to the same equivalence class.

Corollary. An exponent $\xi$ of $G$ whose restriction to the subgroup $\Gamma$ is equivalent to zero, has restrictions equivalent to zero for all subgroups $\Gamma_{g}=g \Gamma g^{-1}$ conjugated to $\Gamma$. This follows at once from (4).

Proposition 2. An exponent of $G$ whose restriction to the subgroup $\Gamma$ is equivalent to zero, is equivalent to zero on $G \times \Gamma$. That is to say, if

$$
\xi\left(\gamma_{2}, \gamma_{1}\right)=\zeta\left(\gamma_{2}\right)+\zeta\left(\gamma_{1}\right)-\zeta\left(\gamma_{2} \gamma_{1}\right) \quad\left(\gamma_{1}, \gamma_{2} \in \Gamma\right)
$$

then, there exists a function $\eta$ on $G$ such that

$$
\xi(g, \gamma)=\eta(g)+\eta(\gamma)-\eta(g \gamma) \quad(g \in G, \gamma \in \Gamma) .
$$

The converse is obvious.

Proof. Consider the left cosets $G / \Gamma$ and choose a representative $h$ in each one, so that each group element $g$ can be written: $g=h(g) \delta(g)$, where $h(g) \in G \mid \Gamma, \delta(g) \in \Gamma$. Obviously, $h(g \gamma)=h(g)$ and $\delta(g \gamma)=\delta(g) \gamma$, $\forall \gamma \in \Gamma$. According to (5) now:

$$
\begin{aligned}
\xi(g, \gamma) & =\xi(h(g) \delta(g), \gamma) \\
& =\xi(\delta(g), \gamma)+\xi(h(g), \delta(g) \gamma)-\xi(h(g), \delta(g)) \\
& =\zeta(\delta(g))+\zeta(\gamma)-\zeta(\delta(g \gamma))+\xi(h(g \gamma), \delta(g \gamma))-\xi(h(g), \delta(g))
\end{aligned}
$$

which proves $(6)$, with

$$
\eta(g)=\zeta(\delta(g))-\xi(h(g), \delta(g)) .
$$

Accordingly, if $\xi$ is an exponent of $G$ whose restriction to $T$ is equivalent to zero, one may choose another equivalent exponent $\bar{\xi}$ which is equal to zero on $G \times \Gamma .^{2}$

Proposition 3. Two equivalent exponents of $G, \xi$ and $\xi^{\prime}$, whose restrictions to the subgroup $\Gamma$ are both equal to zero, define a one-dimensional representation of $\Gamma$ (not necessarily unique).

Proof. Since $\xi^{\prime}\left(g_{2}, g_{1}\right)=\xi\left(g_{2}, g_{1}\right)+\zeta\left(g_{2}\right)+\zeta\left(g_{1}\right)-\zeta\left(g_{2} g_{1}\right)$, and $\xi\left(\gamma_{2}, \gamma_{1}\right)=\xi^{\prime}\left(\gamma_{2}, \gamma_{1}\right)=0, \forall \gamma_{2}, \gamma_{1} \in \Gamma$, then $\zeta\left(\gamma_{2} \gamma_{1}\right)=\zeta\left(\gamma_{2}\right)+\zeta\left(\gamma_{1}\right)$.

${ }^{2}$ This proof is incomplete in that it implicitly assumes the existence of a continuous representative $h(g)$, which is not necessarily true. Our results thus only apply in cases where this condition is fulfilled. Happily, this is the case for all physical examples to be studied (it is trivially true when $G$ is a semi-direct product $X$ (8) $\Gamma$, for instance). I will come back to this question in a forthcoming paper. It is a pleasure to thank Prof. J. A. Dieudonné for drawing my attention to this point. 


\section{$2 c$}

Definition. Two exponents of $G, \xi$ and $\xi^{\prime}$, whose restrictions to $\Gamma$ are equivalent to zero, are said to be $\Gamma$-superequivalent if they are equivalent (on $G$ ) and if the equivalence of the two exponents $\bar{\xi}$ and $\bar{\xi}^{\prime}$, respectively equivalent to $\xi$ and $\xi^{\prime}$ and equal to zero on $\Gamma$, may be realized by a function equal to zero on $\Gamma$ (the trivial representation of $\Gamma$ ):

$$
\bar{\xi}^{\prime}\left(g_{2}, g_{1}\right)=\bar{\xi}\left(g_{2}, g_{1}\right)+\theta\left(g_{2}\right)+\theta\left(g_{1}\right)-\theta\left(g_{2} g_{1}\right)
$$

with $\theta(\gamma)=0, \forall \gamma \in \Gamma$. This is obviously an equivalence relation.

Proposition 4. Two exponents of $G$, $\xi$ and $\xi^{\prime}$, whose restrictions to $\Gamma$ are equivalent to zero, are $\Gamma$-superequivalent if and only if the one-dimensional representation of $\Gamma$, defined by their respective equivalent exponents $\bar{\xi}$ and $\bar{\xi}^{\prime}$ equal to zero on $\Gamma$, may be extended to a one-dimensional representation of the group $G$.

Proof. According to Proposition 3, the two equivalent exponents $\bar{\xi}$ and $\bar{\xi}^{\prime}$, zero on $\Gamma$, define a function $\xi$ on $G$ whose restriction to $\Gamma$ is a representation of $\Gamma$ :

$$
\bar{\xi}^{\prime}\left(g_{2}, g_{1}\right)=\bar{\xi}\left(g_{2}, g_{1}\right)+\zeta\left(g_{2}\right)+\zeta\left(g_{1}\right)-\zeta\left(g_{2} g_{1}\right) .
$$

If $\xi$ and $\xi^{\prime}$ are $\Gamma$-superequivalent, Eq. (8) holds, which, combined with (9) gives

$$
\zeta\left(g_{2} g_{1}\right)-\theta\left(g_{2} g_{1}\right)=\zeta\left(g_{2}\right)-\theta\left(g_{2}\right)+\zeta\left(g_{1}\right)-\theta\left(g_{1}\right)
$$

In other words, $\omega(g)=\zeta(g)-\theta(g)$ is a one-dimensional representation of $G$, whose restriction to $\Gamma$ is identical to $\zeta$ (since $\theta=0$ on $\Gamma$ ).

Conversely, if there exists a one-dimensional representation $\omega$ of $G$, whose restriction to $\Gamma$ is equal to $\zeta$, the function $\theta=\zeta-\omega$ is such that (10) holds and is zero on $\Gamma$. From (9) one then infers (8), and $\xi$ and $\xi^{\prime}$ are $T$-superequivalent.

It results from Proposition 4 that each equivalence class of exponents of $G$ equivalent to zero on $\Gamma$ may be split into classes of $\Gamma$-superequivvalence characterized by the one-dimensional representations of $\Gamma$ which cannot be extended to a one-dimensional representation of $G$.

Example. When $G$ is a semi-direct product $X \odot \Gamma$, or more generally when $G$ is homomorphic to $\Gamma$, any representation of $\Gamma$ is a representation of $G$, so that there is only one class of $\Gamma$-superequivalence for each equivalence class of exponents equivalent to zero on $\Gamma$.

\section{Gauge Functions}

\section{3a. Definitions and Notations}

Let $X$ be the space-time continuum and $G$ its group of kinematical automorphisms, acting transitively on $X$. We denote by $x \rightarrow g x$ the action of a group element $g$ on the space-time points $x$. For the sequel, 
it is sufficient to think of $X$ as a homogeneous space of $G$, to be identified with a space of left cosets, that is the quotient $G \mid \Gamma$ of $G$ by one of its subgroups $\Gamma$. The stabilizer $\Gamma_{x}$ of a point $x \in X$, i.e. the subgroup of $G$ leaving $x$ invariant (the "little group" of $X$ ), is isomorphic to $\Gamma$. The stabilizers $\Gamma_{x}$ and $\Gamma_{y}$ of any two points $x, y \in X$ are conjugate subgroups, such that $\Gamma_{y}=k^{-1} \Gamma_{x} k$ where $k$ is any element of $G$ such that $k y=x$.

The action of a material point (we shall say a "particle") is a realvalued continuous function $A\left(x_{1}, x_{2}\right)$ on $X \times X$ of the form:

$$
A\left(x_{1}, x_{2}\right)=\int_{s_{1}}^{s_{2}} L\left(x(s), \frac{d x}{d s}\right) d s,
$$

where $x(s)$ is the trajectory of the particle ${ }^{3}$, i.e. an application $\mathbb{R} \rightarrow X$, with end-points $x_{1}=x\left(s_{1}\right), x_{2}=x\left(s_{2}\right)$.

$L$ is the corresponding Lagrangian:

$$
L\left(x, \frac{d x}{d s}\right)=\lim _{x^{\prime} \rightarrow x}\left[\frac{\partial}{\partial x^{\prime}} A\left(x, x^{\prime}\right)\right] \cdot \frac{d x}{d s} .
$$

The real trajectory followed by a particle from $x_{1}$ to $x_{2}$ corresponds to an extremal value of the action functional.

Two actions $A$ and $A^{\prime}$ are equivalent, that is lead to the same equations of motion, if there exists a function $\lambda$ on $X$ such that:

$$
A^{\prime}\left(x_{1}, x_{2}\right)=A\left(x_{1}, x_{2}\right)+\lambda\left(x_{2}\right)-\lambda\left(x_{1}\right),
$$

for the variational problem (with fixed end-points) then is unchanged. Correspondingly, the two Lagrangians $L$ and $L^{\prime}$ are equivalent if they differ by a perfect differential:

$$
L^{\prime}\left(x, \frac{d x}{d s}\right)=L\left(x, \frac{d x}{d s}\right)+\frac{d}{d s} \lambda(x) .
$$

Accordingly, for the equations of motion of a particle to be invariant under the group $G$, the action must transform as follows:

$$
A\left(g x_{1}, g x_{2}\right)=A\left(x_{1}, x_{2}\right)+\alpha\left(g ; x_{2}\right)-\alpha\left(g ; x_{1}\right)
$$

where $\alpha$ is a real function on $G \times X$. Note that $\alpha(e ; x)$ is a constant on $X$ which may always be chosen equal to zero. It follows from (12) that the Lagrangian transform as:

$$
L\left(g x, \frac{d}{d s} g x\right)=L\left(x, \frac{d x}{d s}\right)+\frac{d}{d s} \alpha(g ; x(s)) .
$$

A function on $X \times X$ transforming as in (15) will be called a gaugevariant function, and $\alpha$ will be called a gauge function, or simply a gauge, of the group $G$ for its homogeneous space $X$.

${ }^{3}$ Note that $x(s)$ is a trajectory in the whole space-time, a world-line, parametrized by an arbitrary "evolution parameter" $s$. 
A gauge function $\alpha$ is said to be equivalent to zero if it is of the form:

$$
\alpha(g ; x)=\phi(x)-\phi(g x)+\chi(g),
$$

where $\phi$ is some function on $X$ and $\chi$ a function on $G$. Indeed, for such a gauge, the action transforms as:

$$
A\left(g x_{1}, g x_{2}\right)=A\left(x_{1}, x_{2}\right)+\phi\left(x_{2}\right)-\phi\left(g x_{2}\right)-\phi\left(x_{1}\right)+\phi\left(g x_{1}\right),
$$

so that one can choose another equivalent action [see (13)],

$$
A_{0}\left(x_{1}, x_{2}\right)=A\left(x_{1}, x_{2}\right)+\phi\left(x_{2}\right)-\phi\left(x_{1}\right)
$$

which is invariant under $G$ :

$$
A_{0}\left(g x_{1}, g x_{2}\right)=A_{0}\left(x_{1}, x_{2}\right) .
$$

Finally, two gauge functions will be called equivalent if they differ by a gauge function equivalent to zero.

The gauge functions of the group $G$ for its homogeneous space $X$ form a real vector space. One is interested in fact in the quotient space of this vector space by the above equivalence relation, that is the vector space of equivalence classes of gauge functions.

Choosing a conventional origin $x_{0}$ in $X$, it will be convenient to work with $x_{0}$-centered gauge functions as representatives of their equivalence class, that is gauges obeying

$$
\alpha\left(g ; x_{0}\right)=0 .
$$

This choice may be achieved by the equivalence transformation $\alpha(g ; x) \rightarrow \alpha(g ; x)+\chi(g)$, where $\chi(g)=-\alpha\left(g ; x_{0}\right)$.

We now investigate the group-theoretical properties of gauge functions.

\section{3b. Properties of Gauge Functions}

Lemma. A gauge function $\alpha$ of $G$ for $X$ obeys the following identity

$$
\alpha\left(g^{\prime} ; g x\right)+\alpha(g ; x)-\alpha\left(g^{\prime} g ; x\right)=\xi\left(g^{\prime}, g\right),
$$

where $\xi$, a function on $G \times G$, independent of $x$, is an exponent of $G$ equivalent to zero on $T$.

Proof. i) As a consequence of associativity for the group law, the action $A$ must transform in the same way under the sequence of operations $g$ and $g^{\prime}$ as under the single operation $g^{\prime} g$. This requires the compatibility of:

and

$$
A\left(g^{\prime} g x_{1}, g^{\prime} g x_{2}\right)=A\left(x_{1}, x_{2}\right)+\alpha\left(g^{\prime} g ; x_{2}\right)-\alpha\left(g^{\prime} g, x_{1}\right)
$$

$$
\begin{aligned}
A\left(g^{\prime} g x_{1}, g^{\prime} g x_{2}\right)= & A\left(g x_{1}, g x_{2}\right)+\alpha\left(g^{\prime} ; g x_{2}\right)-\alpha\left(g^{\prime} ; g x_{1}\right) \\
= & A\left(x_{1}, x_{2}\right)+\alpha\left(g ; x_{2}\right)+\alpha\left(g^{\prime} ; g x_{2}\right) \\
& -\alpha\left(g ; x_{1}\right)-\alpha\left(g^{\prime} ; g x_{1}\right) .
\end{aligned}
$$


That is to say:

$$
\begin{aligned}
\alpha\left(g^{\prime} ; g x_{2}\right)+ & \alpha\left(g ; x_{2}\right)-\alpha\left(g^{\prime} g ; x_{2}\right) \\
& =\alpha\left(g^{\prime} ; g x_{1}\right)+\alpha\left(g ; x_{1}\right)-\alpha\left(g^{\prime} g ; x_{1}\right)
\end{aligned}
$$

or :

$$
\alpha\left(g^{\prime} ; g x\right)+\alpha(g ; x)-\alpha\left(g^{\prime} g ; x\right)=\xi\left(g^{\prime}, g\right),
$$

independently of $x$, since $x_{1}$ and $x_{2}$ in (25) are arbitrary points in $X$.

ii) This property implies that:

$$
\begin{aligned}
\xi\left(g^{\prime \prime}, g^{\prime} g\right)+ & \xi\left(g^{\prime}, g\right)-\xi\left(g^{\prime \prime} g^{\prime}, g\right) \\
= & \alpha\left(g^{\prime \prime} ; g^{\prime} g x\right)+\alpha\left(g^{\prime} g ; x\right)-\alpha\left(g^{\prime \prime} g^{\prime} g ; x\right) \\
& +\alpha\left(g^{\prime} ; g x\right)+\alpha(g ; x)-\alpha\left(g^{\prime} g ; x\right) \\
& -\alpha\left(g^{\prime \prime} g^{\prime} ; g x\right)-\alpha(g ; x)+\alpha\left(g^{\prime \prime} g^{\prime} g ; x\right) \\
= & \alpha\left(g^{\prime \prime} ; g^{\prime} y\right)+\alpha\left(g^{\prime} ; y\right)-\alpha\left(g^{\prime \prime} g^{\prime} ; y\right) \\
= & \xi\left(g^{\prime \prime}, g^{\prime}\right)
\end{aligned}
$$

where $y=g x$. Also: $\xi(e, e)=\alpha(e ; x)=0$. The function $\xi$ thus satisfies the defining identities (1) of an exponent of $G$.

iii) Let $\gamma \in \Gamma_{y}$. By definition then, $\gamma y=y$, so that

$$
\xi(g, \gamma)=\alpha(g ; y)+\alpha(\gamma ; y)-\alpha(g \gamma ; y),
$$

and the exponent $\xi$ is equivalent to zero on $G \times \Gamma_{y}$ (take $\zeta(g)=\alpha(g ; y)$ ). This is true for any point $y \in X$, so that, in conformity with the Corollary to Proposition $1, \xi$ is equivalent to zero for the whole family of conjugate subgroups $\Gamma_{y}$, isomorphic to the subgroup $\Gamma$ such that $X=G \mid \Gamma$.

Main Theorem. There is a one-to-one correspondence between the equivalence classes of gauge functions of a group $G$ for its homogeneous space $X=G \mid \Gamma$, and the classes of $\Gamma$-superequivalence of exponents of $G$ equivalent to zero on $\Gamma$.

$A$ canonical realization of the correspondence is contained in the following proof.

Proof. i) We choose a conventional origin $x_{0}$ in $\mathrm{X}$, and work, from now on, with $x_{0}$-centered gauges as representatives of their equivalence class [see (21)]. Further, let $x \rightarrow h_{x}$ be an application $X \rightarrow G$ such that

$$
h_{x} x_{0}=x \text {. }
$$

The $h_{x}$ are a set of representatives of the cosets $G / \Gamma_{x_{0}}$.

ii) From (22), one now has:

$$
\xi\left(g^{\prime}, g\right)=\xi\left(g^{\prime}, h_{g x_{0}}\right)=\alpha\left(g^{\prime} ; g x_{0}\right) .
$$

Let $\alpha$ and $\alpha^{\prime}$ be two equivalent gauges, that is obeying:

$$
\alpha^{\prime}(g ; x)=\alpha(g ; x)-\phi(g x)+\phi(x)+\chi(g) .
$$


If both $\alpha$ and $\alpha^{\prime}$ are $x_{0}$-centered:

$$
0=\phi\left(x_{0}\right)-\phi\left(g x_{0}\right)+\chi(g),
$$

so that one may also write:

$$
\alpha^{\prime}(g ; x)=\alpha(g ; x)+\phi(x)-\phi\left(x_{0}\right)-\left[\phi(g x)-\phi\left(g x_{0}\right)\right] .
$$

The exponents $\xi$ and $\xi^{\prime}$ defined by $\alpha$ and $\alpha^{\prime}$ according to (30) are equal to zero on $G \times \Gamma_{x_{0}}$ and related by

$$
\begin{aligned}
\xi^{\prime}\left(g^{\prime}, g\right) & =\xi\left(g^{\prime}, g\right)+\phi\left(g x_{0}\right)-\phi\left(x_{0}\right)-\phi\left(g^{\prime} g x_{0}\right)+\phi\left(g^{\prime} x_{0}\right) \\
& =\xi\left(g^{\prime}, g\right)+\zeta(g)+\zeta\left(g^{\prime}\right)-\zeta\left(g^{\prime} g\right),
\end{aligned}
$$

where

$$
\zeta(g)=\phi\left(g x_{0}\right)-\phi\left(x_{0}\right)
$$

When restricted to $\Gamma_{x_{0}}$, obviously $\zeta=0$, so that $\xi^{\prime}$ and $\xi$ are $\Gamma_{x_{0}}$-superequivalent.

iii) Conversely, let $\xi$ be an exponent of $G$ equal to zero on $\Gamma_{x_{0}}$. The formula

$$
\alpha(g ; x)=\xi\left(g, h_{x}\right)
$$

defines an $x_{0}$-centered gauge function, obeying (22) with the same $\xi$. Let $\xi$ and $\xi^{\prime}$ be two equivalent exponents equal to zero on $G \times \Gamma$, i.e. satisfying

$$
\xi^{\prime}\left(g_{2}, g_{1}\right)=\xi\left(g_{2}, g_{1}\right)+\zeta\left(g_{2}\right)+\zeta\left(g_{1}\right)-\zeta\left(g_{2} g_{1}\right)
$$

with $\xi$ a one-dimensional representation of $\Gamma$, obeying also

$$
\zeta(g)+\zeta(\gamma)=\zeta(g \gamma) \quad \forall g \in G, \gamma \in \Gamma .
$$

The gauge functions $\alpha$ and $\alpha^{\prime}$ they define are related by:

We may write

$$
\alpha^{\prime}(g ; x)=\alpha(g ; x)+\zeta(g)+\zeta\left(h_{x}\right)-\zeta\left(g h_{x}\right) .
$$

$$
g h_{x}=h_{g x} \gamma(g, x) \quad \text { where } \quad \gamma(g, x)=h_{g x}^{-1} g h_{x} \in \Gamma_{x_{0}} .
$$

Then, according to (38)

so that

$$
\zeta\left(g h_{x}\right)=\zeta\left(h_{g x} \gamma(g, x)\right)=\zeta\left(h_{g x}\right)+\zeta(\gamma(g, x)),
$$

$$
\alpha^{\prime}(g ; x)=\alpha(g ; x)-\zeta(\gamma(g, x))+\phi(x)-\phi(g x)+\chi(g),
$$

where $\chi(g)=\zeta(g)$ and $\phi(x)=\zeta\left(h_{x}\right)$. In other words, $\alpha^{\prime}(g ; x)$ is equivalent to $\bar{\alpha}(g ; x)$ :

$$
\bar{\alpha}(g ; x)=\xi\left(g ; h_{x}\right)-\zeta\left(h_{g x}^{-1} g h_{x}\right) .
$$

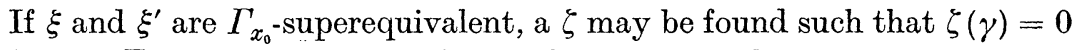
for $\gamma \in \Gamma_{x_{0}}$, so that $\bar{\alpha}=\alpha$, and $\alpha^{\prime}$ and $\alpha$ are equivalent.

If $\xi$ and $\xi^{\prime}$ are not $\Gamma_{x_{0}}$-superequivalent, then formula (43) gives an explicit construction of all the gauge functions of $G$ for $X$ starting from any exponent $\xi$ equal to zero on $G \times \Gamma_{x_{0}}$ and any one-dimensional representation $\zeta$ of $\Gamma_{x_{0}}$ which cannot be extended to $G$ 


\section{Examples}

In the following, we will frequently use the notation $\dot{x}=d x / d s$.

\section{4a. Relativistic Classical Mechanics}

$G$ is the Poincaré group $\mathscr{P}$ with elements noted $g=(a, \Lambda), \Gamma$ is the homogeneous Lorentz group $\mathscr{L}$ with elements $\gamma=(0, \Lambda)$, and $X$ is the Minkowski space $\mathscr{M}$ with elements $x=(t, \boldsymbol{r})$. $G$ acts on $X$ according to $(a, \Lambda) x=\Lambda x+a$. Since the Poincaré group has no non-trivial exponents $[4,10]$ and the Lorentz group has no non-trivial one-dimensional representations, there are only trivial, equivalent to zero, gauges of $\mathscr{P}$ for $\mathscr{M}$ and the action, as well as the Lagrangian, may always be chosen to be strictly invariant under $\mathscr{P}$. According to (16):

or

$$
L\left(\Lambda x+a, \frac{d}{d s}(\Lambda x+a)\right)=L\left(x, \frac{d x}{d s}\right)
$$

$$
L(\Lambda x+a, \Lambda \dot{x})=L(x, \dot{x}) .
$$

Invariance under translations requires $L$ not to depend on $x$ (choose $a=-\Lambda x$ ), and invariance under Lorentz transformations requires $L$ to depend only on the Minkowskian length $\dot{\tau}$ of the four-vector velocity $\dot{x}=(\dot{t}, \dot{\boldsymbol{r}})$. Finally, since $L d s$ must be a first order infinitesimal, $L$ is homogeneous of degree $(+1)$ in the derivatives $(\dot{t}, \dot{\boldsymbol{r}})$ so that

$$
L=-m \dot{\tau}=-m \sqrt{\dot{t}^{2}-\dot{\boldsymbol{r}}^{2}},
$$

where $m$ is an arbitrary real number. Obviously, the possible choice $s=t$ gives back the standard formulation [2]. The progress here is to have rigorously justified the use of an invariant action.

\section{Nonrelativistic Classical Mechanics}

$G$ here is the Galilei group $\mathscr{G}$ with elements $g=(b, \boldsymbol{a}, \boldsymbol{v}, R), \Gamma$ the homogeneous Galilei group with elements $\gamma=(0,0, \boldsymbol{v}, R)$ and $X$ the Newtonian space-time with elements $x=(t, \boldsymbol{r})$, on which $\mathscr{G}$ acts according to :

$$
\left.\begin{array}{l}
t^{\prime}=t+b \\
\boldsymbol{r}^{\prime}=R \boldsymbol{r}+\boldsymbol{v} t+a
\end{array}\right\}
$$

The Galilei group now has non-trivial exponents, which may be chosen in the form $[4,7,11]$ :

$$
\xi\left(g^{\prime}, g\right)=m\left(\frac{1}{2} \boldsymbol{v}^{\prime 2} b+\boldsymbol{v}^{\prime} \cdot R^{\prime} \boldsymbol{a}\right),
$$

where $m$ is an arbitrary real number. These exponents are seen to be equal to zero on $G \times \Gamma$ (that is for $\boldsymbol{a}=0, b=0$ ), and so are admissible for our purposes. Since the homogeneous Galilei group does not have non-trivial one-dimensional representations, the equivalence classes of 
gauge functions are uniquely specified by the equivalence classes of exponents that is by the number $m$. With the natural choice

$$
h_{(t, r)}=(t, r, 0, I)
$$

we obtain the gauge functions:

$$
\alpha(g ; x)=m\left(\frac{1}{2} \boldsymbol{v}^{2} t+\boldsymbol{v} \cdot R \boldsymbol{r}\right) .
$$

The transformation properties of the Lagrangian are then, using (16):

$$
\begin{aligned}
L[(R \boldsymbol{r}+\boldsymbol{v} t+\boldsymbol{a}, t+b), & (R \dot{\boldsymbol{r}}+\boldsymbol{v} \dot{t}, \dot{t})] \\
= & L[(\boldsymbol{r}, t),(\dot{\boldsymbol{r}}, \dot{t})]+m\left(\frac{1}{2} \boldsymbol{v}^{2} \dot{t}+\boldsymbol{v} \cdot R \dot{\boldsymbol{r}}\right) .
\end{aligned}
$$

Choosing first $(b, \boldsymbol{a}, \boldsymbol{v}, R)=(-t,-\boldsymbol{r}, 0, I), L$ is seen to depend only on the velocities $(\dot{t}, \dot{\boldsymbol{r}})$ because of translation invariance. Putting now $(b, \boldsymbol{a}, \boldsymbol{v}, R)=(0,0,-R \dot{\boldsymbol{r}} / i, R)$, galilean invariance is seen to imply:

$$
L(\dot{t}, \dot{\boldsymbol{r}})=L(\dot{t}, 0)+\frac{1}{2} m \frac{(\dot{\boldsymbol{r}})^{2}}{\dot{t}} .
$$

But, since the Lagrangian must be a homogeneous function of first degree in the derivatives:

$$
L(\dot{t}, 0)=k \dot{t}=\frac{d}{d s}(k t),
$$

where $k$ is an arbitrary number. This perfect differential can be eliminated, so that we obtain a Lagrangian

$$
L=\frac{m}{2} \frac{(\dot{\boldsymbol{r}})^{2}}{\dot{t}}
$$

which is the standard result, at least when one chooses $s=t(\dot{t}=1)$. Here again, the progress consists only in proving that it is perfectly safe to remember the possibility of gauge variance of the Lagrangian only when it can no longer be forgotten, that is for pure Galilean transformations [3]. No restriction is implied by choosing a Lagrangian strictly invariant under space-time translations and rotations. Finally, it is instructive to compare the transformation properties of the classical action, given by the gauge function (50), and of the phase of the quantal wave function $[4,5,7]$ : they are identical, in conformity with the known relationship between these two concepts.

\section{4b. One-Dimensional Kinematics ${ }^{4}$}

We now survey the various one-dimensional classical mechanics corresponding to different kinematical groups of interest [12]. These

4 The two-dimensional kinematics offer nothing new compared to the threedimensional cases studied above, although some care must be exercised in the Galilean case: the two-dimensional Galilei group has a covering group with a threedimensional vector space of equivalence classes of exponents. Nevertheless, the only exponents of the group itself which are equivalent to zero on $\Gamma$, are of the same form (48) as in the three-dimensional case. 
related but distinct cases will enable us to exhibit the full richness of the structures studied in the preceding section and their comparison will be most instructive.

The same notation will be used in the various cases. The elements of the group $G$ will be noted $g=(b, a, v), b$ being the parameter for the time translations, $a$ for the space translations and $v$ for the pure inertial transformations, the relation of which to the space-time translations characterizes the group structure. $\Gamma$ is the homogeneous group $\{(0,0, v)\}$ and $X$ the space-time continuum with points $x=(t, r)$, such that one may choose $h_{x}=(t, r, 0)$ as representatives of the left cosets $G / \Gamma$. Finally, the Lie algebra of $G$ has a base consisting of $(H, P, K)$, respectively the generators for time translations, space translations and pure inertial transformations.

One-Dimensional Poincaré Group. The group law reads

$\left(b^{\prime}, a^{\prime}, v^{\prime}\right)(b, a, v)=\left(b^{\prime}+c h v^{\prime} b+s h v^{\prime} a, a^{\prime}+c h v^{\prime} a+s h v^{\prime} b, v^{\prime}+v\right)$

corresponding to the Lie brackets

$$
[H, P]=0, \quad[K, H]=i P, \quad[K, P]=i H .
$$

There exists a one-dimensional family of non-trivial exponents, equivalent to zero on $T$ however, corresponding to the infinitesimal extension of the Lie algebra ${ }^{5}$

$$
[H, P]=i f I,
$$

where $I$ belongs to the center of the extended algebra and the real number $f$ characterizes the extension. Integrating this relation, the exponent may be put in the form:

$$
\xi\left(g^{\prime}, g\right)=f\left[s h^{2} v^{\prime} a b+\frac{1}{2} s h v^{\prime} c h v^{\prime}\left(b^{2}+a^{2}\right)+a^{\prime}\left(c h v^{\prime} b+s h v^{\prime} a\right)\right] \text {. }
$$

The one-dimensional representations of $\Gamma$ obviously extends to $G$, since it is a semi-direct product $X \odot \Gamma$. Hence the $\Gamma$-superequivalence classes of exponents are characterized by $f$, and we may write the most general gauge function as

$$
\alpha(g ; x)=f\left[s h^{2} v t r+\frac{1}{2} \operatorname{sh} v \operatorname{ch} v\left(t^{2}+r^{2}\right)+a(\operatorname{ch} v t+\operatorname{sh} v r)\right] .
$$

To obtain the Lagrangian which must transform according to (16), we note that:

$$
\begin{aligned}
\frac{d}{d s} \alpha(g ; x(s)) & =f\left[\operatorname{sh}^{2} v(\dot{t} r+t \dot{r})+\operatorname{sh} v \operatorname{ch} v(t \dot{t}+r \dot{i})+a(\operatorname{ch} v \dot{i}+\operatorname{sh} v \dot{r})\right] \\
& =f(\operatorname{chv} r+\operatorname{sh} v t+a)(\operatorname{chv} \dot{t}+\operatorname{sh} v \dot{r})-f r \dot{t} \\
& =\beta\left(g x, \frac{d}{d s} g x\right)-\beta\left(x, \frac{d x}{d s}\right)
\end{aligned}
$$

${ }^{5}$ For details concerning the infinitesimal characterization of exponents of Lie groups, see Ref. [4]. 
where

$$
\beta\left(x, \frac{d x}{d s}\right)=f r \dot{t} .
$$

It results from $(60)$ and (16), that $L(x, \dot{x})-\beta(x, \dot{x})$ is invariant under $G$. The same arguments as in the three-dimensional Poincaré case now apply. Finally, we obtain the Lagrangian

$$
L=-m \sqrt{\dot{i}^{2}-\dot{r}^{2}}+f r \dot{t}
$$

which is not the Lagrangian for a "free" particle, but describes a particle moving in a constant force field $f^{6}$ ! This proves that in the customary Lagrangian formalism, "free" motion is not necessarily specified in a unique way by the requirements of invariance under the appropriate kinematical group (see Section 5).

One-Dimensional Galilei Group. One has here:

and

$$
\left(b^{\prime}, a^{\prime}, v^{\prime}\right)(b, a, v)=\left(b^{\prime}+b, a^{\prime}+a+v^{\prime} b, v^{\prime}+v\right)
$$

$$
[H, P]=0, \quad[K, H]=i P, \quad[K, P]=0 .
$$

The vector space of equivalence classes of exponents is two-dimensional. Corresponding to the extended Lie algebra characterized by the two real numbers $(m, f)$ :

$$
[H, P]=\text { if } I, \quad[K, P]=\mathrm{i} m I,
$$

one may write the exponent in the form

$$
\xi\left(g^{\prime}, g\right)=m\left(\frac{1}{2} v^{\prime 2} b+v^{\prime} a\right)+f\left(\frac{1}{2} v^{\prime} b^{2}+a^{\prime} b\right)
$$

These are equal to zero on $G \times \Gamma$, and since $G$, as above, is a semi-direct product $X \odot \Gamma$, one obtains the most general gauge function:

$$
\alpha(g ; x)=m\left(\frac{1}{2} v^{2} t+v r\right)+f\left(\frac{1}{2} v t^{2}+a t\right) .
$$

The same procedure as above yields the Lagrangian

$$
L=\frac{1}{2} m \frac{\dot{r}^{2}}{\dot{t}}+f r \dot{t}
$$

describing the non-relativistic motion of a particle with mass $m$ in a constant force field $f$.

One-Dimensional Newton (or Oscillator) Group ${ }^{7}$.

$$
\begin{aligned}
& \left(b^{\prime}, a^{\prime}, v^{\prime}\right)(b, a, v) \\
& \quad=\left(b^{\prime}+b, a^{\prime} \cos b+v^{\prime} \sin b+a, v^{\prime} \cos b+a^{\prime} \sin b+v\right)
\end{aligned}
$$

${ }_{6}$ Of course, this constant field may be eliminated by requiring invariance under space reflexions for instance.

7 This less known kinematical group, its structure, physical meaning and relations to more usual groups, are studied in Ref. [12] for the three-dimensional case. We limit ourselves here to the "elliptic" case, the "hyperbolic" one being completely similar. 
and

$$
[H, P]=-i K, \quad[K, H]=i P, \quad[K, P]=0 .
$$

The group has a one-dimensional family of exponents corresponding to the extension

$$
[K, P]=\mathrm{i} m I
$$

of the Lie algebra ${ }^{8}$. They may be written as:

$\xi\left(g^{\prime}, g\right)=m\left[\frac{1}{2}\left(a^{\prime 2}+v^{\prime 2}\right) \operatorname{sh} b \operatorname{ch} b+a^{\prime} v^{\prime} \operatorname{sh}^{2} b+\left(v^{\prime} \operatorname{ch} b+a^{\prime} \operatorname{sh} b\right) a\right]$.

A new feature however is that the homogeneous group $T=\{(0,0, v)\}$ has one-dimensional representations:

$$
\zeta(v)=f v
$$

( $f$ a real number), which cannot be extended to the whole group $G$, and formula (43) must be used to obtain the most general gauge function. An easy computation shows that

$$
\gamma(g ; x)=h_{g x}^{-1} g h_{x}=(0,0, v c h t+a s h t) .
$$

Our gauge functions then read:

$$
\begin{aligned}
\alpha(g ; x)= & m\left[\frac{1}{2}\left(v^{2}+a^{2}\right) \operatorname{sh} t c h t+a v \operatorname{sh}^{2} t+(v \operatorname{ch} t+a \operatorname{sh} t) r\right] \\
& +f(v c h t+a s h t) .
\end{aligned}
$$

The Lagrangian is obtained by the methods already used:

$$
L=\frac{m}{2}\left(\frac{\dot{r}^{2}}{\dot{t}}+r^{2} \dot{t}\right)+f r \dot{t} .
$$

Once more, it describes a particle subjected to the constant force field $f$, although in an oscillating universe (or, more trivially, attached to a spring).

One-Dimensional de Sitter Group. We will not give full details here, since the explicit formulas are more complicated than in the preceding cases. The general situation however is easily grasped. The Lie algebra is:

$$
[H, P]=-i K, \quad[K, H]=i P, \quad[K, P]=i H .
$$

The group being simple, it has no nontrivial exponents [4]. The onedimensional representations of the homogeneous group, characterized by a real number $f$ [as in (73)], cannot be extended to the whole group and yield a one-dimensional family of $\Gamma$-superequivalent exponents, hence of gauge functions. The Lagrangian has an invariant part characterized by a real number $m$ and a gauge-variant one characterized by $f$.

8 It may be noted that the group is isomorphic to the one-dimensional Poincare group (substitute $K \rightarrow-H, H \rightarrow K$ ), so that one may use the previous results on the exponents of the one-dimensional Poincaré group. 
Summary of the One-Dimensional Cases. In the four cases studied, the most general Lagrangian yielding invariant equations of motion describes a particle with mass $m$ in a constant force field $f$. These identical physical situations however have very different mathematical descriptions since the parameters $(m, f)$ may characterize group invariants, group exponents or group representations:

de Sitter: $m, G$-invariant $f, T$-one-dimensional representation ,

Poincaré: $m, G$-invariant $f, G$-exponent ,

Newton: $m, G$-exponent $\quad f, \Gamma$-one-dimensional representation ,

Galilei: $\quad m, G$-exponent $\quad f, G$-exponent .

A more detailed study of the contraction processes relating these various groups might be interesting in connection with the different characterizations of their associated invariant motions. Anyway, we have shown with these examples that all the aspects of the general theory developed in Section 3 might find concrete illustrations.

\section{Conclusions}

We have been able to give a complete group-theoretical study of the transformation properties of one-particle Lagrangians in classical mechanics. It should be said, however, that these results may still be recast in a more concise mathematical formalism borrowing some notions from cohomology theory for instance.

But a more interesting problem seems to be posed by one result of the present investigation. It is very strange indeed that requiring the equations of motion to be invariant under some kinematical group does not lead uniquely to inertial motions according to the basic group law. Let us elaborate a bit upon this point taking the one-dimensional Galilei group as an example. It is pretty clear that motion in a constant force field, leading to a quadratic equation of motion, $r=F(t)$, is invariant under the group in the sense that the substitution $\left(r^{\prime}=r+v t+a, t^{\prime}\right.$ $=t+b$ ) yields a new equation of motion $r^{\prime}=F^{\prime}\left(t^{\prime}\right)$, equally quadratic and corresponding to the same constant acceleration, $\frac{d^{2} r}{d t^{2}}=\frac{d^{2} r^{\prime}}{d t^{\prime 2}}$, that is to the same force field. But, after all, a similar situation would prevail for any polynomial equation of motion. And, finally, it is obvious that any rigid motion may be consistently described by various galilean observers. The privileged role of motion in a constant field then appears somewhat arbitrary. Obviously, it is due to the differential equation of motion being of second-order, so that a constant as well as a zero acceleration give an invariant equation. This is not a very satisfying "explanation" however. In fact, it is perhaps possible to give a formulation of classical mechanics, slightly more general but not less natural 
than the customary one, in which the only invariant motions, in a Lagrangian formalism, are given by the inertial motions of the kinematical group. This question is under current investigation.

It is a pleasure to thank H. Ekstein, R. Hakim, M. Le Bellac and J. P. Provost for stimulating conversations.

\section{References}

1. Wrgner, E. P.: Physics Today 17, 34 (1964).

2. Landau, L. D., and E. M. Lifshitz: Field Theory. § 2.2. London: Pergamon Press 1959.

3. - - Classical mechanics. $\S \S 3,4$. London: Pergamon Press 1958.

4. Bargmann, V.: Ann. Math. 59, 1 (1954).

5. Landau, L. D., and E. M. Lifshitz: Quantum mechanics. §15. London: Pergamon Press 1965.

6. Lévy-Leblond, J. M.: Commun. Math. Phys. 6, 286 (1967).

7. - J. Math. Phys. 4, 776 (1963).

8. Pauri, M., and G. Prosperi: J. Math. Phys. 7, 366 (1966).

9. Tassie, L. J., and H. A. Buchdahl: Australian J. Phys. 17, 431 (1964); 18, 109 (1965).

10. Wrgner, E. P.: Ann. Math. 40, 149 (1939).

11. Bernstein, H. J.: J. Math. Phys. 8, 406 (1967).

12. BACRY, H., and J. M. LÉvy-Leblond: J. Math. Phys. 9, 1605 (1968).

J.-M. LÉVY-LEBLOND

Laboratoire de Physique Théorique

Faculté des Sciences

Parc Valrose

F 06 - Nice 